PLY-MS-99-91

\title{
Hadrons Without Strings
}

\author{
Martin Lavelle' and David McMullan'] \\ School of Mathematics and Statistics \\ The University of Plymouth \\ Plymouth, PL4 $8 A A$ \\ $U K$
}

\begin{abstract}
Descriptions of hadrons and glueballs can be constructed using strings to preserve gauge invariance. We show how this string dependence may be removed at all orders in perturbation theory.
\end{abstract}

\footnotetext{
${ }^{1}$ email: mlavelle@plymouth.ac.uk

${ }^{2}$ email: dmcmullan@plymouth.ac.uk
} 


\section{Introduction}

Gauge invariant descriptions of hadrons and their substructures are needed to directly link phenomenology to QCD. The need for gauge invariance becomes clear when one thinks of jet formation: here a quark and an anti-quark move away from each other and turn into two independent jets of hadrons. To obtain such colourless objects, without making the quark matter directly interact so that jet formation becomes implausible, one needs to surround the quarks with glue, which makes the system locally gauge invariant. It is then energetically favoured for this glue to split into two as the quark matter fields reach a large enough separation. This is naively described by parton fragmentation [1] or, closer to QCD, modelled by a gluonic string snapping [2]. In reality, of course, the glue will have a much richer structure.

It is known [3] that colour charge is only defined on locally gauge invariant states and thus all physically meaningful hadronic substructures - coloured quarks in jet formation, constituent quarks, the form of the glue in glueballs and the pomeron - must be described in a gauge invariant way. The appropriate variables for short and long distance physics will vary, however, there are far too few methods known with which they can be described. In this paper we want in particular to show how to proceed from string-based to constituent-type descriptions. We will argue that these latter variables provide a better starting point for probing the above structures.

Non-local states and currents which are supposed to overlap with hadrons are generally made gauge invariant with the insertion of 'strings', i.e., the fundamental fields are linked by path ordered exponentials. To illustrate this, consider the case of Quantum Electrodynamics (QED) where we can make a candidate description of positronium by attaching a string that runs from one fermion to the other over a path $\Gamma$ as depicted in Fig. 11.

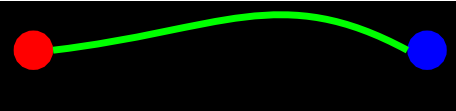

Figure 1: Using strings to try to describe $e^{+} e^{-}$states.

This system (which we for simplicity keep in a single time slice)

$$
\bar{\psi}(t, \boldsymbol{x}) \exp \left\{-i e \int_{\Gamma} d z_{i} A_{i}(t, \boldsymbol{z})\right\} \psi(t, \boldsymbol{y})
$$

is gauge-invariant, however, it has a manifest path-dependence which has no obvious physical interpretation. It is, though, possible to remove this path dependence using 
the standard decomposition of $A_{i}$ into longitudinal $\left(A_{i}^{L}\right)$ and gauge-invariant, transverse $\left(A_{i}^{T}\right)$ degrees of freedom: $A_{i}=A_{i}^{L}+A_{i}^{T}$ where $A_{i}^{L}=\partial_{i} \partial_{j} A_{j} / \nabla^{2}$. We so obtain

$$
\bar{\psi}(x) \mathrm{e}^{i e \frac{\partial_{i} A_{i}}{\nabla^{2}}(x)} \mathrm{e}^{-i e \int_{\Gamma} d z_{i} A_{i}^{T}(t, \boldsymbol{z})} \mathrm{e}^{-i e \frac{\partial_{i} A_{i}}{\nabla^{2}}(y)} \psi(y)
$$

where $\nabla^{-2}$ is the standard Green's function for the spatial Laplacian.

Note that by writing the positronium state in this way we have put all of the arbitrary $\Gamma$-dependence into a separately gauge invariant factor and it is natural to drop it. The remaining gauge-invariant combination

$$
\bar{\psi}_{D}(x) \psi_{D}(y):=\bar{\psi}(x) \mathrm{e}^{i e \frac{\partial_{i} A_{i}}{\nabla^{2}}(x)} \mathrm{e}^{-i e \frac{\partial_{i} A_{i}}{\nabla^{2}}(y)} \psi(y)
$$

is a product of two individually gauge invariant terms, $\psi_{D}$, which were first written down by Dirac [4 and have been employed by a variety of authors in the intervening period (for a review, see [5]).

Further strong physical reasons for dropping the path dependent factor in (2) can be seen by considering the energy of the (straight line) string state. To find the expectation value of the Hamiltonian, $\mathcal{H}=\frac{1}{2} \int d^{3} w\left(E_{i}^{2}(w)+B_{i}^{2}(w)\right)$, for such states, we employ the standard equal-time commutators, $\left[E_{i}(\boldsymbol{x}), A_{j}(\boldsymbol{y})\right]=i \delta_{i j} \delta(\boldsymbol{x}-\boldsymbol{y})$. The magnetic field commutes with (11) but the electric field does not. We straightforwardly find for the potential energy of this state [6]

$$
V(r)=\frac{e^{2}}{2} \delta_{\perp}^{(2)}(0) r
$$

where $r=|\boldsymbol{x}-\boldsymbol{y}|$ and we have dropped the divergent self-energy contributions (the $r$ independent terms). This shows a linear, confining potential with a divergent coefficient - in QED! It is clear that 'confinement' here is an artefact due to trapping the electric flux in a string and that the divergence of the coefficient is a result of the string being infinitely thin (the $\perp$ subscript indicates that this divergent factor originates from the directions orthogonal to the path of the string).

Of course this, although gauge invariant, is not physical. It corresponds to an infinitely excited state and is not stable [7]. A discussion of this instability and an animation of the decay of such a state can be found at http://www. ifae.es/ ${ }^{\sim}$ roy/qed.html. We can gain further insight from recalling that Gauss' law tells us that $\partial_{i} E_{i}=e \rho$, where $\rho$ is the charge density. If we now decompose the electric field into transverse and longitudinal components, the contribution of the electric field to the above Hamiltonian can be so re-expressed as a sum of two positive semi-definite terms, $\frac{1}{2} \int d^{3} w\left(E_{i}^{T^{2}}+E_{i}^{L^{2}}\right)$. The integral over the longitudinal part of the electric field is evidently fixed by Gauss' law to be the same for any gauge invariant description of a state with a fixed number of 
charges. However, the transverse component, $E_{i}^{T}$, is not so fixed. For the above 'stringy' state it in fact diverges, while for the variable $\psi_{D}$ it is zero which minimises the energy. Hence the stringy state is unstable and it decays into a product of two charged fields as given in (3). It is easy to repeat the analysis leading to (4) for these constituents and so see that the familiar potential of electrodynamics, $-e^{2} / 4 \pi r$, holds for this state. This implies that in any string description of this $e^{+} e^{-}$state, where we can factor out the exponential over the $A_{i}^{T}$ variables, we indeed ought to drop this factor and work with a product of two $\psi_{D}$ variables.

The string-based description has, we have seen, many unattractive characteristics which make it a poor starting point to analyse positronium. The alternative, constituent picture has a much more appealing physical base. Its domain of validity is for heavy charges since the charges, $\psi_{D}$, have to be static [5]. To approach a more realistic description, one would need to expand in the masses and so build in binding energies. However, since this starting point is energetically favoured and has a clear physical interpretation it is a more sensible starting point for studying bound states in QED.

The question now arises, can a similar decomposition be carried out for QCD? The string picture, since the matter fields would feel confining interaction at short distances, does not include the short distance forces felt by quarks and gluons. However, the generalisation of $\psi_{D}$ would include the Coulombic type of interaction which dominates at short distances. It is not, however, initially obvious that this can be done. The vector boson fields are now Lie algebra valued and any $T^{a}$ matrices sandwiched between spinors cannot be extracted. Furthermore the decomposition into longitudinal and gauge-invariant, transverse degrees of freedom used above does not hold in QCD. In the rest of this paper we will show that these obstacles can in fact be overcome at any order in perturbation theory and the path dependence can be removed in QCD. In the mesonic case we will thus regain the constituent structure which has been previously shown [8] by us to be responsible for asymptotic freedom.

\section{Non-Abelian Strings}

We now want to recall how to introduce into a non-abelian theory variables which are both gauge invariant and do not have a path dependence. Then we will show the relation between these degrees of freedom and the string-based systems at lowest order in the coupling.

We define $h^{-1}$ to be a field dependent element of the gauge group that transforms as

$$
h^{-1}(x) \rightarrow h^{-1}(x) U(x) .
$$

Recalling how fermions transform under gauge transformations, $\psi \rightarrow U^{-1} \psi$, it is clear that the following fields

$$
\psi_{h}(x)=h^{-1} \psi(x),
$$


describe gauge-invariant quarks. This is the non-abelian analogue of $\psi_{D}$. We call $h^{-1}$ a dressing, it corresponds here to the gluonic cloud which surrounds a static fermion.

To make this explicit, we now recall that there is a simple generalisation of (3) to all orders in perturbation theory (see the appendix of [5] for a discussion of how to generate it). Essentially we have

$$
h^{-1}=\exp \left\{\left(g \chi_{1}^{a}+g^{2} \chi_{2}^{a}+g^{3} \chi_{3}^{a}+\cdots\right) T^{a}\right\}
$$

where

$$
\chi_{1}^{a}=\frac{\partial_{j} A_{j}^{a}}{\nabla^{2}}, \quad \chi_{2}^{a}=f^{a b c} \frac{\partial_{j}}{\nabla^{2}}\left(\chi_{1}^{b} A_{j}^{c}+\frac{1}{2}\left(\partial_{j} \chi_{1}^{b}\right) \chi_{1}^{c}\right), \quad \text { etc } .
$$

Here $\chi_{1}^{a}$ is, up to a trivial colour factor, what we had above in QED while $\chi_{2}^{a}$ etc are minimal extensions whose role is to preserve the gauge transformation property, Eq. 5 .

In a previous letter [8] we have demonstrated that precisely this gluonic configuration in $h^{-1}$ is responsible for antiscreening. We now want to show how we can use these descriptions to remove the string dependence in the QCD equivalent of Fig. 1:

$$
\bar{\psi}(x) \mathrm{P} \exp \left(g \int_{x}^{y} d z_{i} A_{i}(z)\right) \psi(y),
$$

which is gauge invariant but path dependent. At lowest order in perturbation theory in QCD we can make the same decomposition of the vector potential, since $A_{i}^{T}$ is gauge invariant up to terms of order $g$. We can thus rewrite (9) as

$$
\bar{\psi}(x) h(x) \mathrm{P} \exp \left(g \int_{x}^{y} d z_{i} A_{i}^{T}(z)\right) h^{-1}(y) \psi(y)+O\left(g^{2}\right),
$$

where $h^{-1}$ is the static dressing. We cannot now factor off the exponential due to the presence of the Gell-Mann matrices. However, if we write out the last equation more explicitly:

$$
\bar{\psi}(x)\left(1-g \frac{\partial_{i} A_{i}^{a} T^{a}}{\nabla^{2}}(x)\right)\left[1+g \int_{x}^{y} d z_{i} A_{i}^{T}(z)\right]\left(1+g \frac{\partial_{i} A_{i}^{a} T^{a}}{\nabla^{2}}(y)\right) \psi(y)+O\left(g^{2}\right),
$$

then, since at this order the terms in the square bracket are gauge invariant, we may discard the path dependent term, keeping only the 1, to obtain, at this lowest order, a result equivalent to that which we would obtain from multiplying two dressed quarks, $\psi_{h}$, together. Eq. 11 is suggestive of how this will generalise to higher orders. We stress again that the term which we drop has a singular energy since the flux is concentrated onto a line.

Before moving on to the general proof, let us consider the baryonic case at $O(g)$. Essentially we want to show that the structure of Fig. 20 can be rewritten in pertur- 


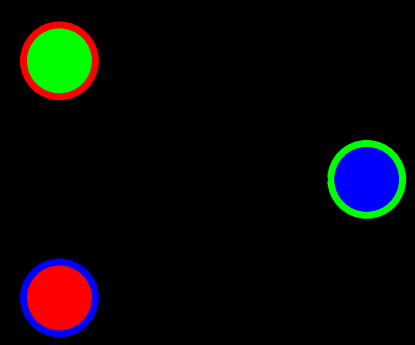

(a)

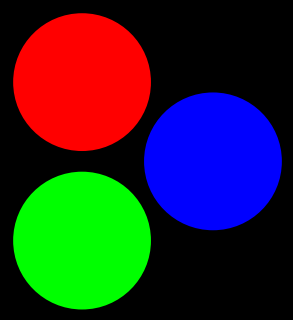

(b)

Figure 2: Two descriptions of a baryon: a) using strings; b) in terms of constituents. Note that a) also possesses a dependence on the point where the strings meet and that only in $b$ ) are there locally gauge invariant substructures with well-defined colour.

bation theory as a product of three gauge invariant quarks, each then with well-defined colour, plus extra additive terms which contain the (singular) path dependence and should be dropped.

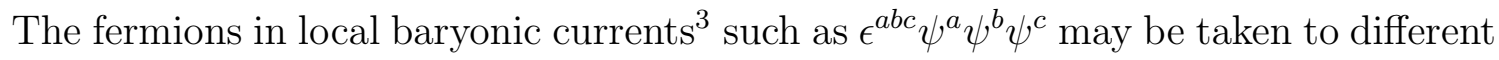
positions in a gauge invariant way by attaching strings as follows

$$
\begin{gathered}
{\left[\mathrm{P} \exp \left(g \int_{w}^{x} d z_{i} A_{i}(z)\right) \psi(x)\right]^{a}\left[\mathrm{P} \exp \left(g \int_{w}^{y} d z_{i} A_{i}(z)\right) \psi(y)\right]^{b}} \\
\times\left[\mathrm{P} \exp \left(g \int_{w}^{l} d z_{i} A_{i}(z)\right) \psi(l)\right]^{c} \epsilon^{a b c} .
\end{gathered}
$$

If we decompose the vector potentials once more into transverse and longitudinal components we obtain at $O(g)$

$$
\begin{aligned}
& {\left[h(w)\left(1+g \int_{w}^{x} d z_{i} A_{i}^{T}(z)\right) h^{-1}(x) \psi(x)\right]^{a}} \\
& \times\left[h(w)\left(1+g \int_{w}^{y} d z^{\prime}{ }_{j} A_{j}^{T}\left(z^{\prime}\right)\right) h^{-1}(y) \psi(y)\right]^{b} \\
& \quad \times\left[h(w)\left(1+g \int_{w}^{l} d z^{\prime \prime}{ }_{k} A_{k}^{T}\left(z^{\prime \prime}\right)\right) h^{-1}(l) \psi(l)\right]^{c}+O\left(g^{2}\right) \epsilon^{a b c} .
\end{aligned}
$$

Again discarding the singular path dependent terms, we obtain at this order two types of terms: those corresponding to an (antisymmetric) product of three dressed quarks at $x, y$ and $l$ and also terms of the form

$$
g \psi^{a}(x) \psi^{b}(y)\left[\frac{\partial_{i} A_{i}^{T}(w)}{\nabla^{2}} \psi(l)\right]^{c} \epsilon^{a b c}+2 \text { similar },
$$

\footnotetext{
${ }^{3}$ The exact Lorentz structure of the current is unimportant.
} 
which come from expanding the $h(w)$ factors in (13). These terms are not path dependent, but they appear to have an unattractive dependence upon the arbitrary point, $w$, where the strings join. However, we can show that they vanish; to do this rename the dummy indices to extract an overall factor of

$$
\left(T^{\alpha}\right)_{c d} \epsilon^{a b c}+\left(T^{\alpha}\right)_{c b} \epsilon^{a c d}+\left(T^{\alpha}\right)_{c a} \epsilon^{c b d},
$$

which vanishes due to the tracelessness of the Gell-Mann matrices. We thus see that on extracting and discarding the path dependence we may, at lowest order in the coupling, write (12) as a product of three dressed quarks, $\psi_{h}$, each of which has a well-defined colour. This system then depends neither on the arbitrary paths nor on their junction point.

It should be clear that we could also attach a string to an individual quark or connect field strengths to produce non-local descriptions of glue. We will return to these applications below. Now though we wish to show how these arguments can be extended to all orders.

\section{All Orders Strings}

We now want to show how to express path ordered exponentials over the vector potentials of QCD in terms of products of path ordered exponentials over the gauge invariant gluons and the dressings. This will permit us to remove the contour dependence to any order of perturbation theory.

Recall that a path ordered exponential [9] transforms under a gauge transformation as

$$
\mathrm{P} \exp \left(g \int_{x}^{y} d z_{i} A_{i}(z)\right) \rightarrow U^{-1}(x) \mathrm{P} \exp \left(g \int_{x}^{y} d z_{i} A_{i}(z)\right) U(y),
$$

and, since a fermion transforms as $\psi \rightarrow U^{-1} \psi$, we can produce gauge invariant composites by linking quarks together via such strings.

We now want to demonstrate that we can rewrite this at all orders as

$$
\mathrm{P} \exp \left(g \int_{x}^{y} d z_{i} A_{i}(z)\right)=h(x) \mathrm{P} \exp \left(g \int_{x}^{y} d z_{i} A_{i}^{h}(z)\right) h^{-1}(y)
$$

where the external dressing factors are gauge dependent and the central term is a path ordered exponential over the gauge invariant field, $A^{h}$. There are no ordering problems for the glue dressed in this fashion.

It is also clear that (17) transforms as one would expect of a path ordered exponential:

$$
h(x) \mathrm{P} \exp \left(g \int_{x}^{y} d z_{i} A_{i}^{h}(z)\right) h^{-1}(y) \rightarrow U^{-1}(x) h(x) \mathrm{P} \exp \left(g \int_{x}^{y} d z_{i} A_{i}^{h}(z)\right) h^{-1}(y) U(y) .
$$


To prove (17), recall the formal definition of a path ordered exponential [9]

$$
\mathrm{P} \exp \left(g \int_{x}^{y} d z_{i} A_{i}(z)\right)=\lim _{N \rightarrow \infty} \prod_{0}^{N-1}\left[1+g A_{i}(z(k))\left\{z_{i}(k+1)-z_{i}(k)\right\}\right],
$$

where the end points $z(0)$ and $z(N)$ are fixed at $x$ and $y$ respectively.

If we consider one element of this product, which we write as $1+g A_{i}(w)\left\{w_{i}-s_{i}\right\}$, and take $\left\{w_{i}-s_{i}\right\}$ to be small then we want to show that this term can be written using the dressing as

$$
1+g A_{i}(w)\left\{w_{i}-s_{i}\right\}=h(s)\left[1+g A_{i}^{h}(w)\left\{w_{i}-s_{i}\right\}\right] h^{-1}(w) .
$$

If we can show this for one term, then (17) follows by inspection. We write the 1 as $h(s) h^{-1}(s)$ and Taylor expand $h^{-1}(s)$ around $w$. Dropping terms of order $\left\{w_{i}-s_{i}\right\}^{2}$ we so obtain

$$
h(s)\left(1+g\left(h^{-1}(s) A_{i}(s) h(s)-\frac{1}{g}\left(\partial_{i} h^{-1}(s)\right) h(s)\right)\left\{w_{i}-s_{i}\right\}\right) h^{-1}(w),
$$

and using $\left(\partial_{i} h^{-1}(s)\right) h(s)=-h^{-1}(s) \partial_{i} h(s)$, we obtain (20) and have thus demonstrated (17).

As well as this formal all orders proof, we have explicitly checked (17) at higher orders. With this result we will now find it relatively straightforward to extract the string dependence from descriptions of hadrons in a gauge invariant way.

\section{Making Hadrons}

Let us now look at the following in turn: quarks, mesons, baryons and finally glue.

a) A quark with a string attached to it may be written as

$$
\mathrm{P} \exp \left(g \int_{\infty}^{y} d z_{i} A_{i}(z)\right) \psi(y)=h(\infty) \mathrm{P} \exp \left(g \int_{\infty}^{y} d z_{i} A_{i}^{h}(z)\right) h^{-1}(y) \psi(y),
$$

where the string is taken along some path out to infinity. Since for reasons of finite energy we require that our fields vanish at spatial infinity, we have $h(\infty) \rightarrow 1$ and we can also factor out the gauge invariant (but contour dependent) path ordered exponential, leaving ourselves with a dressed quark, $h^{-1}(y) \psi(y)$. This shows how one can proceed from the gauge invariant variables introduced by Mandelstam [10] to those of Dirac.

b) A mesonic structure may be made up by linking two fermions by a string

$$
\bar{\psi}(x) \mathrm{P} \exp \left(g \int_{x}^{y} d z_{i} A_{i}(z)\right) \psi(y)=\bar{\psi}(x) h(x) \mathrm{P} \exp \left(g \int_{x}^{y} d z_{i} A_{i}^{h}(z)\right) h^{-1}(y) \psi(y),
$$


where we again have used (17). If we expand the path ordered exponential in $g$ then only the lowest term, unity, is independent of the string's path. Since by construction the higher order terms are gauge invariant, we can drop them and so obtain the non-local, gauge invariant and path independent system, $\bar{\psi}(x) h(x) h^{-1}(y) \psi(y)$. Note that we here cannot factor out the $\Gamma$ dependence but are free to drop this additive, singular term.

c) For baryonic structures we recall (12) which, using our result (17) and discarding as in the mesonic case all the path dependent terms, can be expressed as

$$
\left[h(w) h^{-1}(x) \psi(x)\right]^{a}\left[h(w) h^{-1}(y) \psi(y)\right]^{b}\left[h(w) h^{-1}(z) \psi(l)\right]^{c} \epsilon^{a b c},
$$

which can be rewritten as

$$
h(w)^{a a^{\prime}} h(w)^{b b^{\prime}} h(w)^{c c^{\prime}}\left[\psi^{h}(x)\right]^{a^{\prime}}\left[\psi^{h}(y)\right]^{b^{\prime}}\left[\psi^{h}(l)\right]^{c^{\prime}} \epsilon^{a b c} .
$$

Since both the entire expression and the individual dressed quarks are by construction gauge invariant, $h(w)^{a a^{\prime}} h(w)^{b b^{\prime}} h(w)^{c c^{\prime}} \epsilon^{a b c}$ must also be gauge invariant. However, we can always choose a gauge such that $h(w)=1$, and so $h(w)^{a a^{\prime}} h(w)^{b b^{\prime}} h(w)^{c c^{\prime}} \epsilon^{a b c}=$ $\delta^{a a^{\prime}} \delta^{b b^{\prime}} \delta^{c c^{\prime}} \epsilon^{a b c}$ in all gauges. Therefore (25) can be written solely in terms of dressed quarks as $\psi_{a}^{h}(x) \psi_{b}^{h}(y) \psi_{c}^{h}(l) \epsilon^{a b c}$.

d) As far as glue is concerned we can similarly link field strengths with path ordered exponentials and take traces. The path dependence can then be extracted just as above. Of course we also have for glue the possibility to just directly combine dressed vector potentials, $A_{\mu}^{h} A^{h^{\mu}}$. Such gauge invariant structures have not previously been studied. Condensates of such structures, non-local, gauge invariant and of dimension 2 could be phenomenological significant; we note that many authors have argued for the importance of $\left\langle A_{\mu}^{2}\right\rangle$ condensates - it seems that the stigma of gauge dependence can be removed from such objects (but see below).

\section{Conclusions}

We have seen in QED that string-like descriptions are singular and that the path dependence can be removed, in a gauge invariant way, to yield the correct lowest energy state for static charges. In QCD we have demonstrated that such path dependence can be removed at all orders of perturbation theory. This opens up new, gauge invariant ways to describe hadrons and their substructure.

Fig. 3 shows three possible ways of describing a meson in a gauge invariant fashion. The approach presented in this paper is closest to Fig. 解 which should describe the physics prior to the onset of confinement. (Note though that the fall-off of our variables is such that they extend out to spatial infinity.) In particular they include the Coulombictype interaction between quarks. It is thus evident that, at short distances, where 


\section{Ormo}

(a)

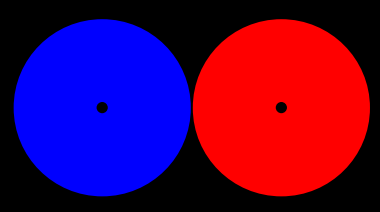

(b)

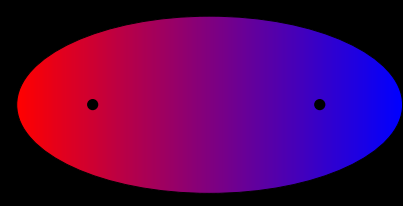

(c)

Figure 3: Three different descriptions of a meson. a) Strings: suitable at strong coupling; b) gauge invariant constituents: describes the short distance glue around current quarks and appropriate before confinement dominates; c) hadronic variables: correct at larger separations.

the forces are essentially Coulombic, the descriptions we suggest are to be preferred. We note that these fields have been shown to describe the glue responsible for antiscreening [8]. It can be shown that the constituent picture presented here does not yet generate three-body forces in baryons. Such forces may emerge from hybrid pictures involving diquarks.

Infinitesimally thin string-type descriptions are only intuitively expected to be relevant in the strong coupling limit, where the shortest path is to be used. (We note that in weak coupling lattice simulations it is found [11] in practice that 'smearing' the string paths in Wilson loop calculations helps in numerical calculations.)

Of course, a physical meson, while certainly not well-described by a string, is not just two constituents and the favoured glue will be in some sense cigar shaped. The emergence of such a picture needs to be further pursued; higher order perturbative studies will be useful in this regard, but non-perturbative work will be crucial. We note here previous attempts to construct, in simpler systems, gluonic distributions which yield a phenomenological potential, of $r+1 / r$ type [12].

There are many questions which immediately cry out to be addressed. The construction presented above holds to all orders of perturbation theory, yet we know that constituent variables only have a limited phenomenological validity. One of the attractive features of our approach is that, although some non-perturbative effects can be incorporated into the construction of the dressing, the construction of $h^{-1}$ is known to break down in the non-perturbative domain [5]. Mapping out its domain of validity is a major focus of our programme. An immediate corollary is that the $\left\langle A^{h} A^{h}\right\rangle$ condensate can only have a limited validity. The physical implications of this fact need to be studied. 
We also note that path-ordered exponentials are widely used in the literature to create non-local, gauge invariant states. We believe that the path independent approach presented here offers an opportunity to test the formalism-dependence of the conclusions of such analyses. From what we have seen above, it seems natural to use these variables as input for variational methods to find the energetically favoured glue in hadronic physics. Finally, the variables used here describe the glue close to current quarks and are suitable for short distance hadronic physics. Parton-hadron duality should we feel be couched in these terms. As such the route from partons to jets will be modified by including dressings and thus these methods have potential implications for jet formation.

Acknowledgements: We thank Emili Bagan, Robin Horan and Shogo Tanimura for discussions.

\section{References}

[1] R. D. Field and R. P. Feynman, Nucl. Phys. B136, 1 (1978).

[2] B. Andersson, G. Gustafson, G. Ingelman, and T. Sjostrand, Phys. Rept. 97, 31 (1983).

[3] M. Lavelle and D. McMullan, Phys. Lett. B371, 83 (1996), hep-ph/9509343.

[4] P. A. M. Dirac, Can. J. Phys. 33, 650 (1955).

[5] M. Lavelle and D. McMullan, Phys. Rept. 279, 1 (1997), hep-ph/9509344.

[6] P. E. Haagensen and K. Johnson, On the wave functional for two heavy color sources in Yang-Mills theory, MIT Report No. MIT-CTP-2614, 1997 (unpublished), hepth/9702204.

[7] L. V. Prokhorov, D. V. Fursaev, and S. V. Shabanov, Theor. Math. Phys. 97, 1355 (1993).

[8] M. Lavelle and D. McMullan, Phys. Lett. B436, 339 (1998), hep-th/9805013.

[9] R. P. Feynman, Phys. Rev. 84, 108 (1951).

[10] S. Mandelstam, Phys. Rev. 175, 1580 (1968).

[11] G. Cella, V. K. Mitryushkin, and A. Vicere, Nucl. Phys. Proc. Suppl. 42, 228 (1995), hep-lat/9412017.

[12] K. Zarembo, Phys. Lett. B421, 325 (1998), hep-th/9710235. 